\title{
Féeries
}

Études sur le conte merveilleux, XVII $-\mathrm{XIX}{ }^{\mathrm{e}}$ siècle

$12 \mid 2015$

À la croisée des genres

\section{Un merveilleux couleur du temps : merveilleux, fantastique et Histoire chez Nodier, Michelet et Sand}

The Marvellous as Period Piece: Marvel, Fantastic and History in Nodier, Michelet and Sand

\section{Paule Petitier}

\section{OpenEdition}

Journals

Édition électronique

URL : http://journals.openedition.org/feeries/961

ISSN : 1957-7753

Éditeur

UGA Éditions/Université Grenoble Alpes

Édition imprimée

Date de publication : 15 octobre 2015

Pagination : 23-37

ISBN : 978-2-84310-306-3

ISSN : 1766-2842

Référence électronique

Paule Petitier, « Un merveilleux couleur du temps : merveilleux, fantastique et Histoire chez Nodier, Michelet et Sand », Féeries [En ligne], 12 | 2015, mis en ligne le 15 octobre 2016, consulté le 08 septembre 2020. URL : http://journals.openedition.org/feeries/961 


\section{UN MERVEILLEUX COULEUR DU TEMPS : MERVEILLEUX, FANTASTIQUE ET HISTOIRE CHEZ NODIER, MICHELET ET SAND}

$A$

$U X I X^{e}$ SIECCLE, la catégorie du fantastique supplante celle du merveilleux. On ne trouve cependant guère à cette époque de théorie sur ce qui distinguerait les deux registres. Il semble qu'il faille plutôt voir dans ce fait de langage l'adoption d'un nouveau point de vue sur le surnaturel : on l'envisage désormais sous l'angle de la subjectivité qui le crée (comme l'indique l'étymologie même du terme "fantastique»), et non comme une surréalité objective - donnée comme telle par la religion - ou une surréalité de convention (telle la fable ou les contes de fées). Le fantastique, chez Charles Nodier par exemple, devient le terme englobant qui désigne toutes les créations de la fantaisie, y compris le merveilleux. Dans la pensée romantique, le merveilleux est fantastique dans la mesure où on estime qu'il est le produit de l'imaginaire collectif des temps archaïques, l'une des formes symboliques qu'il donnait aux faits marquants de la vie sociale. Cependant, le fantastique est aussi considéré dès le $\mathrm{XIX}^{\mathrm{e}}$ siècle comme un genre caractéristique de la modernité, en témoigne par exemple l'article du Grand Dictionnaire universel de Larousse (I866I877) : «Le mot fantastique, mot plus allemand que français, exprime en général des procédés de fabrication littéraire tout modernes.» Cet article oppose le merveilleux, "ancêtre vénérable», à l'innovation du fantastique. Partant de cet embryon d'historicisation qui paraît constituer une sorte de doxa au XIX ${ }^{\mathrm{e}}$ siècle, je voudrais envisager la manière dont trois auteurs, Sand, Nodier et Michelet, en jouent et inscrivent l'antériorité du merveilleux et la modernité du fantastique dans des histoires qui complexifient la relation des deux genres avec l'Histoire. L'enjeu de ces questionnements, qui passent par des formes littéraires plus que par un discours explicite, est sans doute de plaider pour l'invention d'un merveilleux moderne. Nous verrons pourquoi. 


\section{Le fantastique contre l'Histoire}

Les Légendes rustiques de George Sand ont d'abord été publiées de I85I à I855 dans L'Illustration. Tant leurs conditions de publication que la conception de chaque «légende» invitent à considérer cette ouvre comme un recueil, un répertoire de superstitions berrichonnes et de contes dont l'ordre importe peu. Pourtant à l'ouverture du recueil se succèdent trois textes dont il n'est pas permis de douter qu'ils construisent un point de vue historique sur le fantastique. Le premier, «Les Pierres-Sottes ou PierresCaillasses», évoque le caractère archaïque des croyances superstitieuses. À la suite, «Les Demoiselles» illustre par une aventure arrivée à un gentilhomme du XVIII ${ }^{e}$ siècle la croyance aux personnages fantastiques tissés de brume que l'on rencontre dans les lieux marécageux. Le troisième texte, "Les Laveuses de Nuit ou Lavandières", ramène le lecteur au XIX ${ }^{e}$ siècle par le témoignage d'un contemporain de Sand, à qui ces fantômes sont apparus. De la référence aux temps primitifs à celle au temps de l'écriture, une perspective chronologique accélérée se dessine.

Le premier texte évoque le caractère archaïque des croyances liées aux mégalithes ou aux blocs erratiques. Un jeu réflexif entre le discours sur les croyances et l'objet de celles-ci renforce le lien du légendaire et de l'archaïque. En effet les pierres, constate Sand, renvoient elles-mêmes à une histoire géologique très reculée. De surcroît, leur dispersion dans l'espace fait écho à celle du matériau légendaire, le "grand poème de la merveillosité ${ }^{1}$ disséminé dans les traditions orales, dont même la collecte à travers toutes les régions de France ne donnerait qu'une faible idée. Les légendes liées aux pierres constituent selon Sand les traces d'un passé très reculé, elles conservent la mémoire des premières religions supplantées par de nouveaux cultes. Dans la deuxième partie du texte, à propos d'un autre canton du Berry, Sand évoque un autre type d'origine archaïque, que nous appellerions l'animisme primitif. Les superstitions liées aux pierres s'expliqueraient par la croyance des simples à la vie universelle, croyance qui se traduit dans des contes à la tonalité grotesque.

Le deuxième texte, "Les Demoiselles", sans se prononcer sur l'origine de la croyance dans ces personnages fantastiques, l'illustre à une période précise, le "siècle dernier». La tonalité de l'aventure de M. de la Selle, en accord avec son cadre historique, n'est pas sans évoquer le merveilleux du Cabinet des fées. L'existence du surnaturel n'y cause aucun effroi et la

I. G. Sand, Légendes rustiques. Les esprits et les visions de la nuit dans les campagnes [Paris, A. Morel, I858], Paris, Marabout, 1975, p. I5. 
perturbation occasionnée par l'intervention des «demoiselles» est complètement réparée à la fin du conte. Résumons l'histoire : $M$. de la Selle, revenant avec son métayer Luneau de la foire où il a vendu une paire de bœufs, ne trouve plus dans sa valise qu'une poignée de cailloux au lieu de l'argent de la vente. Le métayer suggère que les demoiselles les ont dévalisés alors qu'ils passaient à proximité de l'endroit qu'elles hantent habituellement. M. de la Selle prend son parti de l'incident et refuse de faire assécher la zone marécageuse, comme le voudrait Luneau, pour ne pas déloger les êtres fantastiques que son père lui a recommandé de respecter : "[...] leur présence est un bien dans une terre, et leur protection est un porte-bonheur dans une famille ${ }^{2}$.»Dix années plus tard, alors que Luneau vient de trépasser, M. de la Selle repasse au même endroit en revenant de la foire. Bien éveillé cette fois, il aperçoit les demoiselles et ne peut s'empêcher de les saluer. Elles tentent de l'entraîner dans le marais, mais, sans se démonter, le gentilhomme continue de leur adresser la parole courtoisement et les prie de le laisser aller. Après avoir demandé à M. de la Selle de faire dire une messe pour Luneau, les demoiselles disparaissent. Une fois chez lui le gentilhomme retrouve dans sa valise l'argent dérobé dix ans auparavant. Bien que la rumeur avance une explication rationnelle (une mise en scène du fils de Luneau pour restituer au maître l'argent dérobé), M. de la Selle en reste à la version des demoiselles. Une courtoisie imperturbable est le trait caractéristique du personnage. Le merveilleux est ici, me semble-t-il, clairement rattaché à la fonction de civilisation des mœurs qu'il a prise depuis le $\mathrm{XvII}^{\mathrm{e}}$ siècle $^{3}$. Les ménagements du gentilhomme envers les demoiselles n'ont d'égales que sa bienveillance à l'égard de son métayer (qu'il se refuse à soupçonner). Croyance est ici synonyme de confiance, une confiance dont le caractère naïf ou excessif se voit démenti par un dénouement qui en confirme le bien-fondé. Le merveilleux permet de contourner la violence des rapports sociaux. Une autre de ses caractéristiques est de se ranger du côté du logos (de la raison), comme le montre le fait qu'adresser la parole aux demoiselles — au premier abord une transgression — se retourne en avantage et permet à la Selle d'obtenir sa délivrance.

La troisième «légende» relève d'une tout autre tonalité. Du merveilleux bon enfant on passe au fantastique terrifiant avec «la plus sinistre

2. Ibid., p. 4I.

3. Voir notamment sur ce point les travaux de M. Soriano sur les contes de Perrault et ceux, plus nuancés dans leurs conclusions, de J. Zipes (Les Contes de fées et l'art de la subversion [1983], Payot, 2007 pour la traduction française). 
des visions de la peur ${ }^{4}$ », les lavandières nocturnes. Or cette croyance est illustrée par le récit d'un témoin contemporain de Sand qui, par deux fois, a rencontré en revenant de nuit par des chemins déserts les sinistres lavandières. La réalité du surnaturel est tempérée dans ce texte aussi par la suggestion de causes triviales : l'ébriété du visionnaire, l'intervention de mauvais plaisants. À la différence du texte précédent qui racontait également une histoire arrivée à un individu, le narrateur cède ici la parole au héros de l'aventure, ce qui accentue le caractère subjectif du récit. Autre différence : dans le texte précédent, l'intervention des demoiselles s'intégrait à une histoire (plot) dotée d'une intrigue minimale : un vol suivi d'une restitution. Dans "Les Laveuses de Nuit», rien de tel. L'apparition terrifiante se contente d'accompagner le passant nocturne, sans rien lui faire, sans rien lui demander. Elle se différencie notamment des demoiselles par sa mutité complète. Comme le texte précédent (raison supplémentaire de les comparer) ce récit est en deux temps, l'ami de Sand ayant rencontré deux fois les lavandières. Mais les deux épisodes ne se répondent pas, ils s'ajoutent seulement. Plus aucune communication ne s'instaure avec l'être fantastique. Le surnaturel n'entre plus dans l'ordre du logos, il reste enfermé dans le domaine de l'affect pur. La vision renvoie à une expérience de l'innommable : "[...] son silence, son indifférence à l'approche d'un passant, dit l'homme qui a vu la laveuse, lui donnèrent l'aspect d'un être absolument étranger à notre espèces». La peur et l'interprétation surnaturelle ne se produisent qu'après-coup, presque comme des rationalisations, le moment de la rencontre étant dominé par le dégoût et le malaise. Le choc émotionnel produit par cette vision découle de ce qu'elle met en cause la frontière entre l'objet et le sujet, en renvoyant ce dernier à des angoisses archaïques. Les laveuses de nuit sont réputées être des mères infanticides et ce qu'elles lavent et tordent de leurs mains blêmes ne serait autre que le cadavre de leur enfant. Sand rapproche ensuite plusieurs autres légendes de celle des laveuses de nuit et elles ont toutes à voir avec l'image d'un corps morcelé ou d'une matière mise en lambeaux : il y a la légende des "pieds blancs», qui apparaissent au bord des fossés et des mares sans être rattachés à aucun corps, celle de la brayeuse (broyeuse) de chanvre spectrale, celle de la peillerouse (femme en guenilles) fantôme.

Le fantastique des "Laveuses de Nuit» est serti dans un cadre énonciatif qui lui conferre une tonalité moderne, qui insiste sur sa dimension subjective - non seulement parce que la réalité de la vision est mise en doute, mais parce que la nature de la peur se rattache aux structures du

4. G. Sand, Légendes rustiques, ouvr. cité, p. 47.

5. Ibid., p. 5 I. 
sujet et à l'angoisse de leur ébranlement. Dans leur succession, les trois premières légendes du recueil esquissent donc une évolution : d'abord un fantastique primitif lié aux éléments les plus archaïques du paysage, puis un merveilleux d'Ancien Régime, rattaché au pouvoir civilisateur de la fiction, enfin un fantastique moderne, terrifiant, qui ramène, comme le fantastique primitif, à l'archaïque, mais à un archaïque nettement psychique. La linéarité de ces jalons chronologiques est immédiatement contrebalancée par la correspondance entre le stade le plus ancien et le stade le plus moderne. Ils sont encore liés par un autre point : le thème du morcellement. On le trouve en effet dans le premier texte à travers la dispersion des pierres dans le paysage, reflet de la dissémination du grand poème du merveilleux; on le retrouve dans le fantasme du corps morcelé et dans le commentaire du narrateur sur les histoires analogues à celle des «Laveuses de Nuit»:

Les histoires fantastiques qui ne s'expliquent pas sur la nature des êtres qu'elles mettent en scène, et qui restent vagues et incomplètes, sont celles qui frappent le plus l'imagination ${ }^{6}$.

Le parcours esquissé par les trois premiers textes du recueil ne présente pas la période moderne comme un accomplissement, mais plutôt comme le cadre d'une perte, comme si la vertu sociale et morale du merveilleux des «demoiselles» s'était évanouie. Dans "Les visions de la nuit dans les campagnes", Sand suggère d'ailleurs que l'homme moderne a perdu avec la croyance au merveilleux une façon de se prémunir contre les aberrations de la perception. Tandis que les hommes de la campagne en contact incessant avec la nature ont de fréquentes hallucinations mais les projettent à l'extérieur d'eux et s'en protègent ainsi en les objectivant (sous forme d'êtres surnaturels auxquels ils croient), l'homme cultivé des villes se laisse déstabiliser profondément par les fantaisies de ses sens :

Notre grande terreur, à nous autres, quand le cauchemar ou la fièvre nous présentent leurs fantômes, c'est de perdre la raison, et plus nous sommes certains d'être la proie d'un songe, plus nous nous affectons de ne pouvoir nous y soustraire par un simple effort de la volonté. On a vu des gens devenir fous par la crainte de l'être. Les paysans n'ont pas cette angoisse; ils croient avoir vu des objets réels; ils en ont grand peur; mais la conscience de leur lucidité n'étant point ébranlée, l'hallucination est certainement moins dangereuse pour eux que pour nous ${ }^{7}$.

Le fantastique moderne est donc dangereux, en rien constructif pour le sujet.

6. Ibid., p. 53 .

7. Ibid., p. 184 . 
Ces soupçons vont être confirmés par le rapport qu'il entretient avec l'Histoire, point où il se trouve aussi rencontrer le fantastique primitif. Sand souscrit à la théorie romantique selon laquelle les mythes anciens contiennent sous leur forme fabuleuse des références à des événements historiques. Il faut voir dans les croyances populaires, affirme-t-elle dans l' "Avant-propos" des Légendes rustiques, "une histoire inédite des temps passés ${ }^{8} »$. Cependant, elle ne donne guère d'exemples de décryptage du référent historique des légendes. Cela excède, dira-t-on, le travail et les compétences de la collecteuse qu'elle veut être. Soit. Pourtant, si l'on considère les passages dans lesquels elle avance l'hypothèse d'une signification historique des croyances, on est frappé du caractère particulier de l'Histoire dont les légendes porteraient la trace :

Le blé qui pousse a le pied dans la chair humaine dont la poussière a engraissé nos sillons. Tout est ruine, sang et débris sous nos pas, et le monde fantastique qui enflamme ou stupéfie la cervelle du paysan est une histoire inédite des temps passés?

L’emploi du terme «fantastique», et non de "merveilleux» utilisé également par Sand dans cette œuvre, est ici symptomatique — non seulement de la perspective subjective dans laquelle sont commentées les croyances, mais aussi du rapport à l'Histoire qu'elles impliquent. Le fantastique archaïque semble ne se rapporter qu'à une Histoire violente. Ainsi, l'un des rares cas où Sand ose assigner une cause historique est celui des pierres levées qui conserveraient la mémoire de cultes sanguinaires :

Les sacrifices de victimes humaines semblent planer, comme une horrible réminiscence, dans certaines visions. Les cadavres ambulants, les fantômes mutilés, les hommes sans tête, les bras ou les jambes sans corps, peuplent nos landes et nos vieux chemins abandonnés ${ }^{10}$.

Le fantastique conserve donc essentiellement trace d'une Histoire «noire», malheureuse, refoulée. Une Histoire qui ne passe pas. Les Légendes rustiques suggèrent ainsi que le fantastique recouvre un rapport problématique à l'Histoire. Dans «Les Trois Hommes de Pierre», Sand fait l'hypo-

8. Ibid., p. I6. G. Sand indique plus précisément ses références dans le compte rendu du livre de Chaix : "Grâce à l'école nouvelle dont MM. Littré, Renan et autres éminents écrivains nous ont révélé l'esprit, nous arrivons aujourd'hui à regarder l'histoire des fictions comme l'étude de l'homme même, puisque toute fiction est l'idéalisation d'une impression reçue dans un certain temps et dans un certain milieu historique. Plus on recule dans le passé, plus la fiction tient de place; à ce point même qu'elle est la seule histoire des premiers âges." (Ibid., "Croyances et Légendes du Centre de la France», p. 175)

9. Ibid., p. I5-16.

Io. Ibid., p. I7. 
thèse d'un rapport entre la croyance aux « retournants », esprits condamnés à retourner des pierres dans la Creuse, et l'Histoire récente. "Ces légendes se rattachent, sans doute, au lugubre souvenir des désastres causés par les crues subites et terribles de la rivière ${ }^{\mathrm{II}} »$ qui eurent lieu en $\mathrm{I} 845$. Le lecteur est surpris qu'un fait aussi récent soit déjà devenu légende. À y regarder de plus près, on remarque les particularités du désastre à l'origine de cette immédiate transmutation légendaire : les crues avaient provoqué l'accumulation d'arbres arrachés, de «masses de toits, de bateaux, de barrières et de débris de toutes sortes", monstrueux barrage qui en cédant tout à coup à la pression de l'eau avait «balay[é] tout sur son passage ${ }^{12}$ ». Cette catastrophe naturelle n'est pas sans évoquer les barricades de 1848 encore bien présentes à l'esprit des contemporains au début du Second Empire, moment de l'écriture. Le fantastique apparaît comme un double refoulement de l'Histoire : barricade déguisée en barrage, événement récent immédiatement transposé en légende.

Le surnaturel des Légendes rustiques relève bien, en grande partie, du fantastique et non du merveilleux, parce que, loin d'être une fiction civilisatrice, il recouvre une histoire violente, qui fait retour et traduit une angoisse. La période à laquelle Sand compose ces textes, de I85I à I855, conforte évidemment cette interprétation. Le fantastique populaire révèle un rapport problématique à l'Histoire, son impossibilité, justement. Comment le transformer en un merveilleux progressiste? Voilà sans doute la question à laquelle les Contes d'une grand-mère (I873) apportent plus tard une réponse, en tentant de réconcilier Histoire, formation de l'individu et merveilleux, dans des fables éducatives et progressistes. Ce merveilleux réconcilié avec la raison, destiné également à l'homme rationaliste moderne, renoue avec le merveilleux "Ancien Régime» illustré par «Les Demoiselles» dans les Légendes rustiques, et retrouve la fonction civilisatrice du conte.

\section{De Nodier à Michelet : nature, Histoire et merveilleux}

L'histoire de l'amour impossible entre la belle Jeannie et le lutin d'Argail, sujet du conte de Nodier, Trilby (I822), se déroule en même temps qu'une transformation du merveilleux.

II. Ibid., p. 77 .

I2. Ibid., p. 78 . 
L'ouverture du conte en présentant les croyances du peuple écossais décrit un âge d'or, tout y exprime le bonheur, la joie de vivre, la vitalité. Le lutin, être éminemment mobile et subtil (au sens premier), relie le monde naturel et le monde humain. Grâce à ce personnage merveilleux, qui circule des vieilles aux jeunes femmes, aux vaches, aux chevaux et à l'âtre, s'instaurent un rapport immédiat, une continuité de l'humain et du naturel. Le lutin rend sensible l'érotisme diffus de ce monde innocent dans lequel tout est lié à tout.

[...] il caracole sur les chevaux qui hennissent de joie, roule dans ses doigts les longs anneaux de leurs crins flottants, lustre leur croupe polie, ou lave d'une eau pure comme le cristal leurs jambes fines et nerveuses ${ }^{13}$.

Il n'est pas loin de remplir ainsi la fonction qu'Hésiode assigne à Éros dans la cosmogonie, celle d'une divinité fondamentale pour assurer la cohésion du monde. L'univers peint dans le premier paragraphe du récit est un univers horizontal, sans aucun arrière-monde, et le merveilleux, celui d'un monde entièrement présent.

Or le déroulement du récit amène une inversion totale de cette situation initiale. À ce début solaire répond une fin nocturne, effrayante et macabre, dans le cimetière où l'ermite Ronald, représentant d'un christianisme qui honnit les esprits de la nature, enferme Trilby dans le tronc d'un bouleau, et provoque par là-même la mort de Jeannie. La malédiction lancée contre l'harmonie immanente de la nature transforme le rapport de symbiose initial marqué du sceau du merveilleux en un fantastique inquiétant, où les créatures surnaturelles sont vues comme des causes de trouble et de perdition. La rupture de l'unité primitive se manifeste par deux conséquences. D’une part, le merveilleux est marginalisé, il subsiste dans la description, mais sous une forme purement esthétique. D'autre part, le basculement dans le registre fantastique accompagne l'émergence de la subjectivité.

Après le pèlerinage à l'abbaye de Balva, la batelière contemple de sa barque les rives du lac Beau :

Les vapeurs qui s'élèvent ordinairement sur le lac, et s'étendent au-devant des montagnes sous la forme d'un rideau de crêpe, avaient peu à peu élargi les losanges flottants de leurs réseaux de brouillards. Celles que le soleil n'avait pas encore tout à fait dissipées se berçaient sur l'occident comme une trame d'or tissue par les fées du lac, pour

I3. Ch. Nodier, Contes, éd. P. Castex, Paris, Garnier frères, 1961, p. IO2. 
l'ornement de leurs fêtes. D'autres étincelaient de points isolés, mobiles, éblouissants comme des paillettes semées sur un fond transparent de couleurs merveilleuses ${ }^{\mathrm{I4}}$.

Je ne cite que le début d'une longue pause descriptive célébrant la beauté de la nature. Elle pourrait laisser croire que rien n'a changé depuis l'incipit. Cependant, la mention du «rideau de crêpe» qui a besoin d'être tiré pour dévoiler le paysage suggère bien une mort de la nature. Nous sommes désormais devant une nature mise à distance, convertie en représentation. L'homme en est devenu le pur spectateur. Le merveilleux reste inscrit dans le paysage mais avec le statut de métaphore. La féerie exprime le caractère esthétique de la nature. La nature est belle d'un merveilleux refoulé, sublimé en quelque sorte dans la châsse de cristal du paysage, comme la suite du texte le révèle en rappelant la légende de l'ermite qui a réduit à l'impuissance le géant Arthur installé sur les monts au-dessus du lac.

En parallèle avec cette évolution du rapport de l'homme et de la nature, la forme du texte change rapidement : il commence comme un conte et se poursuit comme un roman, du fait que le personnage de Jeannie rompt avec les caractéristiques essentiellement fonctionnelles du personnage de conte pour se voir doté d'une intériorité tourmentée. À l'incipit évoquant de façon générale la croyance des Écossais aux lutins succède le récit des relations entre un lutin, Trilby, et une jeune femme, Jeannie. Or cette histoire-là se prête à être lue comme celle de la constitution d'une subjectivité féminine. Trilby peut être interprété comme la projection des désirs de Jeannie. Sa situation de femme mariée fait qu'elle éprouve une certaine culpabilité de ces désirs qui n'ont pas pour objet son époux. L'ermite, figure de la loi, sorte d'alter ego du mari, en compagnie duquel il apparaît presque toujours, renforce, en formulant expressément l'interdit, les scrupules de Jeannie et sa division intérieure entre son devoir et son désir. De façon concomitante, le personnage de Trilby évolue. Présent d'abord aux côtés de Jeannie sous la forme d'une créature minuscule, il se transforme par la suite dans les rêves de la batelière en beau jeune homme, ce qui confère à leur relation, toute fantasmagorique qu' elle soit, le caractère d'un adultère. Jeannie manifeste ainsi la constitution d'une subjectivité : l'apparition de la conscience (et donc de la division), les tourments du désir, la culpabilité, mais aussi la rêverie et la compassion. Divisé en luimême, le sujet ne saurait se constituer sans que s'opère aussi une séparation d'avec le monde qui l'entoure; il ne se conçoit comme tel que face au

I4. Ibid., p. I26. 
statut objectif du monde. Il est tout à fait significatif que Trilby, l'esprit de la nature, dans son ultime métamorphose adopte la forme d'un riche coffret. Ainsi le roman de la subjectivité va-t-il de pair avec la transformation du rapport à la nature, son objectivation. La transmutation du merveilleux en objet esthétique est indissociable de l'apparition d'un fantastique lié à la subjectivité. Chassés de la nature les esprits habitent, enrichissent et tourmentent l'intériorité humaine.

Deux passages situés dans l'épisode du pèlerinage au monastère de Balva emblématisent les deux versants de la transformation du merveilleux initial : la description d'un paysage d'une part, le récit d'un incident terrifiant dans l'église même d'autre part.

Après le premier exorcisme, lorsque Ronald a banni Trilby de la chaumière de Dougal et de Jeannie, se produit un premier désenchantement du monde, les objets deviennent inanimés, muets; les choses ne sont plus que des choses. Se rendant au monastère de Balva, Dougal et Jeannie admirent un paysage automnal. Il ne s'agit pas seulement du cycle naturel des saisons : le spectacle exprime un deuil dont l'intensité excède celui d'un flétrissement passager :

On entend sortir du fond des bois une sorte de rumeur menaçante qui se compose du cri des branches sèches, du frôlement des feuilles qui tombent, de la plainte confuse des bêtes de proie que la prévoyance d'un hiver rigoureux alarme sur leurs petits, de rumeurs, de soupirs, de gémissements, quelquefois semblables à des voix humaines, qui étonnent l'oreille et saisissent le cœur. Le voyageur n'échappe pas même à l'abri des temples aux sensations qui le poursuivent ${ }^{15}$.

C'est une véritable scène d'agonie de la nature, qui prend pour le coup des accents fantastiques, avec ces bruits sinistres auxquels on ne peut assigner d'origine claire. La nature morte-vivante parle, mais pour dire son deuil, et sa voix est la dernière manifestation de sa vie, de sa présence, son apparence est déjà spectrale. On est loin d'ores et déjà du merveilleux initial. Les premières phrases de ce tableau décrivent un trompe-l'œil par lequel la nature rappelle sur un mode exacerbé sa sensualité par l'ardeur des couleurs, et à travers l'apparence de la grenade (le grenadier est symbole d'Aphrodite) :

Les feuilles, saisies par le froid matinal, se roulaient à la pointe des branches inclinées, et leurs bouquets bizarres, frappés d'un rouge éclatant, ou jaspés d'un fauve doré, semblaient orner la tête des arbres de fleurs plus fraîches ou de fruits plus brillants que les fleurs et les fruits qu'ils ont reçus de la nature. On aurait cru qu'il y avait des bouquets de grenades dans les bouleaux, et que des grappes mûres pendaient à la

I5. Ibid., p. II4-II5. 
pâle verdure des frênes, surprises de briller entre les fines découpures de leur feuillage léger ${ }^{16}$.

Cependant l'inquiétude domine, du fait de l'étrangeté d'une nature placée désormais du côté de l'illusion, du «comme si», avec ces fruits qui n'en sont pas, ces sons qui imitent des gémissements, et ces mirages qui trompent même les bêtes :

On dirait quelquefois le chant grêle d'une jeune vierge cloîtrée qui répond au mugissement majestueux de l'orgue; et ces impressions se confondent si naturellement en automne, que l'instinct même des animaux y est souvent trompé. On a vu des loups errer sans défiance, à travers les colonnes d'une chapelle abandonnée, comme entre les fûts blanchissants des hêtres $[\ldots]^{17}$.

Non seulement la religion ne protège pas contre les fantasmagories de la nature mourante, mais elle-même semble menacée de ruine comme le suggère la mention de la chapelle abandonnée.

À ce paysage-scène répond le récit donné par Ronald lui-même d'un événement curieux advenu récemment au monastère :

L'autre jour, à l'instant où le cercueil d'un de nos frères allait toucher le sol du caveau mortuaire, la corde se rompt tout à coup en sifflant comme avec un rire aigu, et la châsse roule, grondant, de degrés en degrés [sic] sous les voûtes. Les voix qui en sortaient ressemblaient à la voix des morts, indignés qu'on ait troublé leur sépulture, qui gémissent, qui se révoltent, qui crient. Les assistants les plus rapprochés du caveau, ceux qui commençaient à plonger leurs regards dans sa profondeur, ont cru voir les tombes se soulever et flotter les linceuls, et les squelettes agités par l'artifice des lutins jaillir avec eux des soupiraux, s'égarer sous les nefs, se grouper confusément dans les stalles ou se mêler comme des figures bouffonnes dans les ombres du sanctuaire ${ }^{18}$.

L'offensive des lutins - c'est l'explication que donne Ronald de cet événement mystérieux — n'est pas, on en conviendra, bénigne. Il s'agit presque d'une apocalypse, avec tremblement de terre, résurrection des morts, retour du monde au chaos... Bien pire qu'une apocalypse. $\mathrm{Ne}$ voit-on pas ici la transcendance elle-même, représentée par la lampe du sanctuaire, défaillir?

Toutes les lumières du temple avaient disparu. [...] Enfin la lampe immortelle du Saint des Saints... - Je la vis s'agiter, s'obscurcir, et mourir. Mourir! La nuit profonde, la nuit tout entière, dans l'église, dans le chœur, dans le tabernacle! La nuit descendue pour la première fois sur le sacrement du Seigneur ${ }^{19}$ !
16. Ibid.
I7. Ibid.
I8. Ibid., p. I2O-I2I.
19. Ibid. 
Ce passage situé dans le premier tiers du récit contribue à discréditer l'explication à laquelle une strate apparente du texte pourrait faire croire. La lutte de Ronald contre les esprits païens ne peut plus être lue comme l'affirmation d'une transcendance qui a besoin de nier toute force surnaturelle immanente. Dans ce conte, le christianisme n'est pas plus le vainqueur que le paganisme. Ronald ne représente aucune transcendance, mais seulement sa propre rigidité tyrannique. Il incarne une loi qui n'est plus fondée sur le sacré. N'est-il pas indiqué que le vrai saint, Colombain, est le frère de Trilby? Ils se trouvent d'ailleurs tous les deux réunis au cimetière, l'arbre du saint enserrant dans son tronc le lutin, réunis mais dans l'espace de la mort. On songe, à propos de ce dénouement, à l'interprétation que donne Peter Brooks du fantastique moderne ${ }^{20}$. Celui-ci naîtrait du fait que «les impasses de l'Âge de Raison", à la fin du xvirI ${ }^{\mathrm{e}}$ siècle, ramènent vers le sacré, mais celui-ci, ayant perdu sa transcendance, ne se manifeste plus que «selon les modalités les plus primitives possibles, en tant que tabou et interdit ${ }^{21}{ }^{\prime}$; il ne subsiste du sacré que la terreur, et l'effondrement de la transcendance place les causes de cette terreur dans les profondeurs obscures de la psyché. Aux deux facteurs identifiés par Brooks, insuffisance de la raison, défaillance de la transcendance, il me semble que le conte de Nodier nous invite à ajouter : objectivation de la nature. En effet, les impasses de la raison et la disparition de la transcendance suggèrent à la pensée romantique de se tourner vers un autre champ, celui d'une nature, où pourrait s'ancrer un sacré immanent, et où l'homme pourrait se réassurer ontologiquement. Le merveilleux du début de Trilby décrit ce rêve, comme toutes les évocations romantiques d'un âge d'or païen. Néanmoins, la nature elle-même, prise dans le mouvement irréversible qui depuis la Renaissance l'objective, ne peut guère (re)devenir ce foyer de sens qui unifierait les aspirations humaines. La nature s'en vient donc elle aussi, comme le montre le paysage automnal dans Trilby, alimenter les représentations fantastiques qui traduisent la mise en doute de tous les référents absolus à l'âge moderne.

Le conte de Nodier, on vient de le voir, décrit les apories de la pensée moderne plus qu'il ne les historicise. Cependant, par l'effet même de la mise en intrigue, Trilby invite à reconstruire une histoire du passage du merveilleux au fantastique, dont l'opérateur serait une religion chrétienne hostile à la nature et aux croyances populaires. C'est bien entendu ce qui va intéresser un historien comme Jules Michelet. Il convient tout d'abord

20. P. Brooks, «La Vertu et la terreur : Le Moine», Romantisme, no I62 (4/2013), p. 85-97.

2I. Ibid., p. 85 . 
d'affirmer, à l'encontre de l'opinion courante, la très forte intertextualité qui relie La Sorcière à Trilby. Il me paraît indubitable que le conte de Nodier est la matrice narrative et fantasmatique de La Sorcière (I862). Le rapport entre les deux ouvres a été minimisé ${ }^{22}$ sans doute parce que la critique a fait confiance à un propos condescendant inséré par Michelet dans sa deuxième "Note» : «Le Trilby de Nodier, et la plupart des contes analogues, sont manqués, parce qu'ils ne vont pas jusqu'au moment tragique où la petite femme voit dans le lutin l'infernal amant ${ }^{23}$." Michelet pointe ici en effet son apport personnel, l'infléchissement qu'il donne à l'histoire de Nodier, mais il ne mentionne pas sa dette, pourtant patente. La situation décrite par Nodier au début de son conte, l'intimité indiscrète que la subtilité du lutin lui permet avec la femme, n'a pu que frapper l'imaginaire voyeuriste de Michelet. Au chapitre in de La Sorcière, la description des câlineries que le petit esprit prodigue à la jeune femme réécrit, en en soulignant le côté trouble, le comportement de Trilby avec Jeannie :

Elle est gênée de se sentir suivie partout, s'en plaint et y a plaisir. Parfois elle le renvoie, le menace, enfin se croit seule et se rassure tout à fait. Mais au moment elle se sent caressée d'un souffle léger ou comme d'une aile d'oiseau. Il était sous une feuille... Il rit... ${ }^{24}$

Michelet a perçu d'autre part dans le récit de Nodier un canevas susceptible de soutenir la construction d'une hypothèse historique. J'irai même jusqu'à dire que l'idée de construire la première partie de son œuvre autour du fil conducteur de la vie d'une femme a certainement été inspirée à Michelet par Trilby.

Michelet reprend l'évolution des relations de Trilby et de Jeannie - le fait que cette relation, d'abord innocente et enfantine, devienne progressivement un harcèlement amoureux — et la rattache à des faits historiques. Au haut Moyen Âge, la transformation de l'habitat collectif en habitat dispersé permet la résurgence des anciennes croyances païennes liées à la nature. Voilà qui explique l'apparition des esprits familiers autour de la cabane de la jeune serve. Le durcissement du régime féodal provoque la transformation du lutin indiscret mais inoffensif en esprit obsédant et tentateur, puis la pression de l'État moderne créé par Philippe-le-Bel s'ajoutant à l'oppression seigneuriale déclenche la métamorphose de l'hôte indiscret

22. Ainsi, P. Castex affirme-t-il dans son édition des Euvres de Nodier que malgré la ressemblance des œuvres, J. Michelet «ne songe pourtant pas à Trilby» mais au Monde enchanté (recueil de fables magiques de la fin du XVII ${ }^{\mathrm{e}}$ ), éd. citée, «Notice» du Cycle écossais, p. 9I.

23. J. Michelet, La Sorcière, éd. P. Viallaneix, Paris, Garnier-Flammarion, I966, p. 295.

24. Ibid., p. 67-68. 
en Satan. Michelet amplifie également le roman de la subjectivité : le fantastique de la possession accentue le dédoublement de la conscience, il est ressenti comme la hantise d'un autre dans lequel l'esprit ne parvient plus à reconnaître sa propre création.

La Sorcière de Michelet se bat avec la même aporie que le Trilby de Nodier : l'aspiration à reconstituer un sacré immanent de la nature pour compenser l'effondrement de la transcendance, alors même que la nature est objectivée par l'esprit moderne. Mais là où Nodier en reste au constat d'une double défaillance (ce qui lui vaut la condescendance de Michelet), l'historien tente de refonder à partir du personnage de La Sorcière un sacré immanent. Mon propos n'est ici d'analyser ni la mise en œuvre de cette tentative ni les obstacles contre lesquels en fin de compte elle bute. Je m'en tiendrai à cette constatation qui va dans le droit fil des remarques précédentes : le fantastique est traité par Michelet comme le signe d'un rapport déréglé à l'Histoire et s'inscrit dans sa relecture du Moyen Âge destinée à démontrer qu'une véritable perversion du cours de l'Histoire s'est produite alors ${ }^{25}$. Le fantastique n'est pas seulement relié à la constitution de la subjectivité, il est invoqué pour explorer les déformations imprimées à celle-ci par les systèmes oppressifs lorsque l'Histoire elle-même défaille. Le propos de Michelet fait écho à celui de Sand mais, là où la romancière s'arrêtait au constat du symptôme, le vieil historien s'efforce de donner sens, contenu et figure à la dérive fantastique de l'Histoire.

Qu'en est-il alors du merveilleux? La même chose que du progrès, dont il est l'une des figures ou des promesses. L' "Épilogue ${ }^{26}$ » de La Sorcière évoque l'expérience d'un émerveillement, la contemplation de l'aurore sur la baie de Toulon. Cette merveille naturelle, semblable à la robe couleur du temps de Cendrillon, devient l'emblème d'un merveilleux réparateur tourné vers l'avenir et unissant tout ce qu'il faudrait unir idéalement : religion et science, subjectivité et nature, sens et raison, matière et spiritualité, un merveilleux qui emblématise, au-delà de Michelet, le rêve des penseurs progressistes du XIX ${ }^{\mathrm{e}}$ siècle :

De six à sept, j'avais un moment admirable. La scintillation vive (oserai-je dire acérée?) des étoiles faisait honte à la lune, et résistait à l'aube. [...]

25. À partir du Second Empire, J. Michelet reprend les tomes de l'Histoire de France consacrés au Moyen Âge (écrits sous la Monarchie de Juillet) et les infléchit dans le sens d'une vision extrêmement négative.

26. Ce terme qui désigne « une partie finale ajoutée, comme de surcroît, à un discours, à un ouvrage, en lui-même complet» (G. Vapereau, Dictionnaire universel des littératures, Paris, Hachette, I876, entrée "Épilogue») signifie bien l'extériorité à l'Histoire de cette véritable profession de foi. 
Un bleuâtre indéfinissable (que l'aube rosée respectait, n'osait teinter), un éther sacré, un esprit, faisait toute nature esprit.

On sentait pourtant un progrès, de lents et de doux changements. Une grande merveille allait venir, éclater et éclipser tout ${ }^{27}$.

27. J. Michelet, La Sorcière, ouvr. cité, p. 286. 
